\title{
Intra- and inter-molecular dispersion interactions in $[n]$ Cycloparaphenylenes: Do they influence their structural and electronic properties?
}

J. V. Climent-Medina, A. J. Pérez-Jiménez, M. Moral, E. San-Fabián, and J. C. Sancho-García*

Departamento de Química Física,

Universidad de Alicante, 03080 Alicante, Spain

February 12, 2015

*E-mail: jc.sancho@ua.es 


\begin{abstract}
Cycloparaphenylenes (CPPs) are nanosized structures with challenging isolated and bulk properties. They can be also viewed as synthetic targets for the template-driven (potentially) bottom-up synthesis of carbon nanotubes. Thus, the step by step understanding of the supramolecular order at the nanoscale is of utmost relevance for further molecular engineering. We find here that intra-molecular noncovalent (dispersion) interactions must be taken into account to obtain accurate estimates of structural and optoelectronic properties of these $[n] \mathrm{CPP}$ compounds, analyzing also their influence as the number of repeat units increases from $n=4$ up to $n=12$, both in the gas phase and in solution. We also address the supramolecular self-assembly of [6]CPP, where both intra- and inter-molecular non-covalent interactions are relevant, by calculating the binding energies of dimers taken along several crystal directions. The latter are used also to estimate the cohesive energy of the crystal, which is compared to the value obtained by means of dispersion-corrected DFT calculations using periodic boundary conditions. The reasonable agreement found between both computational strategies points towards a first estimate of the [6] CPP cohesive energy of around $50 \mathrm{kcal} / \mathrm{mol}$.
\end{abstract}




\section{Introduction}

Cycloparaphenylenes (CPPs) represent the shortest sidewall segment of armchair Single-Walled carbon NanoTube (SWNT) structures, and thus have greatly captivated scientists very recently due to their unique architectural features and envisioned possibilities (see Figure 1). The recent achievements concerning the synthesis of these cycloparaphenylenes provide an unique framework for understanding and fully control the template-driven synthesis of fine-tuned nanotubes as envisioned target [1-3]. They also can be viewed as a fragment of buckminsterfullerene structures [4]. For instance, preassembled (tubular but discontinuous) CPPs structures might be further annealed thermally or photochemically to achieve the desired SWNT [5], although the crystalline structure of the former compounds is known to vary from herringbone to tubular packing, without still understanding in depth the reasons of such different behavior. Furthermore, the unusual stability of their cationic and anionic forms might pave the way towards novel synthetic procedures for obtaining aromatic molecules [6].

The twisted and strained para-phenylene units, which close and selfarrange to form the macrocycle in CPPs, allow for radially oriented $\pi$-type electronic interactions, contrarily to linear parent para-phenylenes. This closed configuration induces a large set of new and interesting properties with respect to linear forms [7], such as the diameter of the nanohoop, the strain energy arising from closing these para-phenylenes into a ring, or their necessarily modified electronic and absorption properties. These achievements have attracted recently the interests of several groups [8-17] in an attempt to understand how these properties evolve with system size ( $n$, where $n$ refers to the number of benzene units connected in para position) and how they 
manifest when real samples are obtained in solution or forming solid-state phases. Note that the first cycloparaphenylene was synthesized in 2008 [19], and that there is still a lot of opportunities concerning the rich chemistry of these compounds. However, the subtle interplay between short-, medium-, and long-range electronic effects, as a function of this system size or after some functionalization of the $[n]$ CPP molecules [18], is expected to have some impact on the aforesaid size-dependent properties. This issue needs still to be further studied, and addressed carefully by theoretical methods if one seeks the greatest possible accuracy.

As a matter of example, we summarize next some interesting and highly challenging achievements (in the supramolecular scale) recently accomplished in the field: (i) the encapsulation of $\mathrm{C}_{60}$ by [10]-cycloparaphenylene ([10]CPP), forming a host-guest complex stabilized roughly by $40 \mathrm{kcal} / \mathrm{mol}$ in toluene [20] and with both molecules separated by a distance of $3.35 \AA$, which equals the interlayer separation in graphite; actually, not all the $[n] \mathrm{CPP}$ nanohoops $(n=8-12)$ are able to encapsulate $\mathrm{C}_{60}$ [21], but only [10]CPP, which might arise from the interplay between attractive and repulsive intraand inter-molecular interactions; (ii) the association of $\mathrm{C}_{70}$ or the metallofullerene La@ $\mathrm{C}_{82}$ with [11]CPP was also recently studied $[22,23]$, and shows how both molecules are labile enough to accommodate each other, with [11]CPP adopting an ellipsoidal shape in the former case; (iii) the chirality in SWNTs might be induced by appropriately substituted cycloparaphenylenenaphthalenes [24] or cycloparaphenylene-naphthylenes [25] compounds, constituting thus the first step towards the controlled synthesis of chiroptical SWNTs, which might create a diversity of forms and release the strain energy of unsubstituted cycloparaphenylenes; and (iv) the dimerization of $[n] \mathrm{CPP}$ 
nanohoops, thanks to covalently-linked structures at only one specific position along the macrocycle, which might pave the way towards increasingly longer supramolecular architectures with customized nanochannels [26].

From the set of examples mentioned above, it becomes thus clear how the large interplay between intra- and inter-molecular dispersion (attractive) effects might drive size-dependent properties of these compounds, and will be thereof the main interest of this study. The use of the term 'dispersion' is normally associated with specific long-range inter-molecular non-covalent interactions [27], whose strength peaks at around 3.5-7 $\AA$ and beyond, being normally related to crystal directional growth and supramolecular stability.

However, these effects might have also some influence for isolated molecules such the ones studied here, due to their particular closed form. For this reason, we have chosen to study in depth the [6]CPP case, which has face-to-face opposite benzene units separated by around $6 \AA$, where the above-mentioned intra-molecular interactions might play an important role. On the other hand, the interactions between adjacent benzene units are below that cutoff, and are thus better termed as medium-range interactions. Despite their weaknesses, these interactions cannot be (in principle) neglected even for medium-sized organic molecules, as it has been recently emphasized in order to obtain accurate results [28].

Last but not the least, we want to point out that some trade-offs between accuracy and computational cost will be needed to adequately incorporate the aforementioned interactions, due to the increasing size of the systems studied here. Therefore, we will first describe some theoretical (and techni- 
cal) details before discussing the results for increasingly longer $[n] \mathrm{CPP}$ (individual) systems. Then, we will extend the study to the [6]CPP bulk (mainly in its solid-state) case, which is known to present striking differences with respect to other molecules when it is allowed to supramolecularly organize in real samples. This approach will allow us to disentangle the importance of underlying physical effects on some key properties, as well as to tackle challenging supramolecular issues in this family of interesting compounds.

\section{Theoretical details}

Density Functional Theory (DFT) $[29,30]$ using standard functionals will be employed along the study. More costly methods, scaling with the system size as $O\left(N^{5}\right)$ (with $N$ being related to that size) or beyond, such as $O\left(N^{7}\right)$ Coupled-Cluster Singles Doubles with perturbative Triples $[\operatorname{CCSD}(\mathrm{T})]$, are prohibitive for the larger systems tackled here. Note that the main focus of this work is the possible influence of dispersion interactions, not only as a guideline for further exploiting structure-property relationships but also to bracket their not-yet-studied influence on global properties of these compounds, rather than the benchmarking of theoretical methods and/or density functionals [31-33]. This is why we just consider the BLYP [34,35] and B3LYP [36-38] exchange-correlation functionals, as an example of pure (without exact-like exchange) and hybrid (with a $20 \%$ of exact-like exchange) models respectively, combined with an adequate representation of atomic orbitals (i.e. the $6-31+\mathrm{G}^{*}$ or the family of def2-SVP/def2-TZVP/def2-QZVP basis sets [39]) and with state-of-the-art corrections for dispersion effects; because the latter are expected to play a key role due to the particularities of the systems investigated here, influencing both analytical gradients 
evaluation (i.e. structures) and single-point calculations (i.e. energetics). Although electrostatics and induction interactions can be partially described by the BLYP and B3LYP uncorrected functionals, the prediction of dispersion interactions is still particularly challenging and some corrections have to be customarily introduced. Note also that these systems display some inhomogeneous behavior due to the central vacuum region, and that a complete theoretical (electrodynamics) description would necessitate treatment of many-body effects $[40,41]$, which will be neccessarily truncated and thus approximated here. The approximations followed are described in more detail next:

a) We have addressed these effects by resorting in all cases to the -D3(BJ) method $[42,43]$. We have first incorporated these effects into the DFTbased calculations to correctly tackle the evolution of intra-molecular properties of $[n] \mathrm{CPP}$ with system size, and then the strength of intermolecular interactions (in the case of $[6] \mathrm{CPP}$ ) in a further step. This is effectively done through the function:

$$
E_{\mathrm{D} 3(\mathrm{BJ})}^{(2)}\left(R_{A B}\right)=-\sum_{n=6,8} s_{n} \sum_{B>A}^{\text {atom pairs }} \frac{C_{n}^{A B}}{R_{A B}^{n}+f_{n}\left(R_{A B}^{0}\right)},
$$

where a two-body correction to the electronic energy, $E^{(2)}$, is added as a function of: (i) some functional-dependent parameters $s_{n}$ to efficiently couple both energy, electronic and dispersion, terms; (ii) the averaged (isotropic) $n$ th-order interatomic dispersion coefficients $C_{n}^{A B}$; (ii) all the existing internuclear distances $R_{A B}=\left|R_{A}-R_{B}\right|$; and (iii) the damping function $f_{n}=\left(a_{1} R_{A B}^{0}+a_{2}\right)^{n}$, which allows to switch the energy from medium- to short-distances, with $R_{A B}^{0}=\sqrt{\frac{C_{8}^{A B}}{C_{6}^{A B}}}$ and $a_{1(2)}$ fitted parameters for the functionals used here [44-46]. Note that the older -D2 correction $[47,48]$ is a simplification (a first-version) of the 
above approach, which is however widely and successfully used too. The latter correction will be employed (vide infra) for the crystal treatment of $[6] \mathrm{CPP}$, together with corresponding periodic boundary conditions.

b) When dealing with supramolecularly nanostructured forms (several self-assembled molecules forming a nanoaggregate) of [6]CPP, a threebody correction has been also employed. This is done through the function [49]:

$E^{(3)}\left(R_{A B}, R_{A C}, R_{B C}\right)=\sum_{A>B>C}^{\text {atom triples }} C_{9}^{A B C} \frac{\left(3 \cos \theta_{A B} \cos \theta_{B C} \cos \theta_{A C}+1\right)}{\left(R_{A B} R_{B C} R_{A C}\right)^{3}} f_{n}\left(\bar{R}_{A B C}\right)$,

where $C_{9}^{A B C}$ is the corresponding $n$ th-order interatomic dispersion coefficient, approximated by $C_{9}^{A B C} \approx-\sqrt{C_{6}^{A B} C_{6}^{B C} C_{6}^{A C}}$, and $\theta_{I}$ are the internal angles of the triangle formed by the internuclear distances $R_{A B}-R_{B C}-R_{A C} . \bar{R}_{A B C}$ is the geometric mean of $R_{A B}, R_{B C}$, and $R_{A C}$. The damping function $f_{n}$ also holds a similar form to the one used in Eq. (1). Note that a satisfactory agreement has been recently found between highly accurate $\operatorname{CCSD}(\mathrm{T})$ (and thus many-body) calculations and this correction for trimers of crystalline benzene [50].

c) Another way to incorporate part of the missing non-covalent interactions is to rely on a nonlocal (-NL) correction to the electronic energy in the form:

$$
E_{\mathrm{NL}}^{(2)}=\int d \mathbf{r} \rho(\mathbf{r})\left[\beta(b)+\frac{1}{2} \int d \mathbf{r}^{\prime} \rho\left(\mathbf{r}^{\prime}\right) \Phi\left(\mathbf{r}, \mathbf{r}^{\prime}\right)\right]
$$

with the function $\Phi\left(\mathbf{r}, \mathbf{r}^{\prime}\right)$ coupling the total electronic densities, $\rho(\mathbf{r})$ and $\rho\left(\mathbf{r}^{\prime}\right)$ at two different sampled points of the electronic coordinates space. For the final form of the correction we use the modern construction VV10 [51], and the attenuation parameter $b, \beta=\frac{1}{32}\left(\frac{3}{b^{2}}\right)^{3 / 4}$, as 
obtained from the literature for the BLYP and B3LYP models $[52,53]$ together with the functional selected. Note that we have only selectively used this method as a "sanity check" for some cases, which will be always conveniently indicated.

d) For the larger nanoaggregates of [6]CPP studied here, we have also employed a corrected Hartree-Fock (HF) method. The computational cost is largely reduced by using a small basis set (MINIX) and three corrections $(-3 \mathrm{c})$ to account for missing effects. Thus, the total electronic energy is given by [54]:

$$
E_{\mathrm{HF}-3 \mathrm{c}}=E_{\mathrm{HF} / \mathrm{MINIX}}+E_{\mathrm{D} 3(\mathrm{BJ})}^{(2)}+E_{\mathrm{BSSE}}^{g C P}+E_{c R_{A B}},
$$

where $E_{\mathrm{D} 3(\mathrm{BJ})}^{(2)}$ is merely a re-parameterized version of the expression defined previously, $E_{\mathrm{BSSE}}^{g C P}$ denotes a geometrical counterpoise correction (gCP) to account for basis sets incompleteness issues, and $E_{c R_{A B}}$ corrects the systematically overestimated covalent bond lengths for electronegative elements $A$ and $B$.

As a matter of summary, Table 1 gathers for clarity the various methodologies used in the study reported here, as well as the properties estimated from them.

\section{Results and discussion}

\subsection{Increasingly longer isolated $[n] \mathrm{CPPs}$}

The GAUSSIAN09 [55] package was used for all the calculations described in this part of the study. For the geometry optimization of biphenyl, triphenyl, and the set of $[n] \mathrm{CPP}(n=4-12)$ molecules, the moderate $6-31+\mathrm{G}^{*}$ 
basis set was fixed, thanks to the well-established trade off between accuracy and computational cost and for comparison with previous results found in the literature. Hence, all the geometries were fully optimized at the B3LYPD3(BJ)/6-31+G* level, without symmetry constraints, checking carefully afterwards that all the frequencies were positive, to firmly guarantee in all cases that we have found a true minimun along the hypersurface. Tables 2 and 3 summarize the main results (diameter and strain energy of the nanohoop, and energy difference between frontier molecular orbitals) that will be discussed separately next.

We analyze first two main structural features chosen as example. The first one is the diameter of every $[n] \mathrm{CPP}$, defined as the distance between the opposite ipso-carbon atoms for the even members, or as the distance between the ipso-carbon atom and the center of mass of the opposite benzene ring for the odd members. As it would be expected, the influence of dispersion corrections, at the B3LYP-D3(BJ)/6-31+G* level, is to slightly decrease the diameter in all cases with respect to previously calculated (B3LYP/6-31G*) values [11]. The diameter is found to evolve with system size $(n)$ following the relationship $d=1.379 n-0.011$. The second structural parameter considered is the dihedral angle between adjacent benzene units, that is the $\mathrm{C}_{o^{-}} \mathrm{C}_{i p s o^{-}}$ $\mathrm{C}_{i p s o^{\prime}}-\mathrm{C}_{o^{\prime}}$ angle. Owing to the close relationship between these two structural parameters, diameter and dihedral angles, the values for the latter are expected to concomitantly increase with respect to previously calculated values. This is clearly observed for the even members of $[n] \operatorname{CPP}(n=4,6,8,10,12)$, for which one obtains $\mathrm{C}_{o^{-}} \mathrm{C}_{i p s o^{-}}-\mathrm{C}_{i p s o^{\prime}}-\mathrm{C}_{o^{\prime}}$ values of $21.9^{\circ}, 29.1^{\circ}, 32.4^{\circ}, 34.0^{\circ}$, and $35.1^{\circ}$, respectively, exceeding by $1-2^{\circ}$ the corresponding values predicted before at the B3LYP/6-31G* level [10]. Note that the linear extrapolation 
of this dihedral angle, with respect to the inverse of the system size $(1 / n)$, provides a value of $42^{\circ}$ at the $n \rightarrow \infty$ limit, and thus close to that found for biphenyl [56] as it should be expected when the strain induced by forming the cycle is completely released.

Another interesting property of these nanohoops is their strain energy, rendering them challenging synthetic targets. Strain energies can be evaluated by different ways, among which homodesmotic are probably the best reactions suited for it [57]: Roughly speaking, both the number of each type of $\mathrm{C}-\mathrm{C}$ bond as well as the number of each type of $\mathrm{C}$ atom should be equal in both members of the chemical equation, basically balancing all kinds of stereoelectronic effects. We thus calculate the strain energy of increasingly longer $[n]$ CPPs by means of the expression:

$\Delta H_{\text {strain }}([n] \mathrm{CPP})=n \Delta H_{f}^{\ominus}(p$-triphenyl $)-\left\{\Delta H_{f}^{\ominus}([n] \mathrm{CPP})+n \Delta H_{f}^{\ominus}\right.$ (biphenyl $\left.)\right\}$,

where $\Delta H_{f}^{\ominus}$ refers to the enthalpy (at $0 \mathrm{~K}$ and with the zero-point vibrational energy included) of optimized structures of $p$-triphenyl, $[n] \mathrm{CPP}$, and biphenyl, following the sketch (Scheme 1) used to measure this energy:

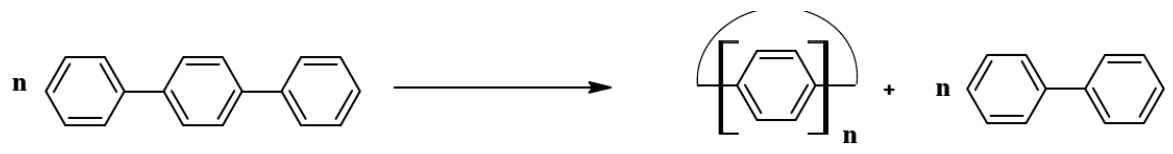

\section{Scheme 1}

Since strain energies are known to pose a challenge to synthetic chemistry, their computational estimate might thus help over synthetic strategies as well as for a better understanding of the inter-molecular interactions dominating the final supramolecular organization of the samples. We next compare in 
Figure 2 the calculated values at the B3LYP-D3(BJ)/6-31+G* level with those obtained at the B3LYP/6-31G* level, the latter taken from Ref. [10]. From the bunch of results obtained, one can state that: (i) the contribution of the dispersion energy, obtained from Eq. (1), to strain energies decreases with system size, as it should be due to the $R_{A B}^{-6}$ decay of weak interactions, roughly ranging (in absolute values) around $\sim 6(\sim 2) \mathrm{kcal} / \mathrm{mol}$ for the smallest (largest) nanohoop studied here; (ii) the weight (in relative values) of this contribution to strain energies remains, however, almost independent of system size due to the observed decrease of their values with system size, roughly ranging between $3.4-4.4 \%$ of the final values; and (iii) the strain energy calculated at the B3LYP-D3(BJ)/6-31+ $\mathrm{G}^{*}$ level evolves with the system size (n) following a power function $A_{0} n^{-A_{1}}$, with $A_{0}=525.0$ and $A_{1}=0.9807$, and a correlation coefficient of 0.9994 .

Figure 2 also shows the difference between the Highest Occupied (HO) and the Lowest Unoccupied (LU) Molecular Orbital (MO) energies, as calculated here at the (single-point) B3LYP/def2-TZVP//B3LYP-D3(BJ)/6$31+\mathrm{G}^{*}$ level, and their comparison with those calculated at the B3LYP/6$31 \mathrm{G}^{*}$ level (taken from Ref. [11]). As the system size increases, the energy of the HOMO decreases (stabilizes) while that of the LUMO increases (destabilizes), so that their energy difference grows accordingly. These counterintuitive results, in sharp contrast with linear oligophenylenes, originate from the size dependence of aromaticity of benzenoid rings, and have been repeatedly shown in the literature and confirmed by Raman spectroscopy [58]. Since the detailed assignment of the UV-vis absorption spectra of these compounds has been also recently done [59], we will exclusively focus in the following on the effect (if any) of the dispersion-corrected geometries on this energy 
gap. Note that, although we have used the large (and then sufficiently polarized) def2-TZVP basis set, the (single-point) results between B3LYP/def2TZVP//B3LYP-D3(BJ)/6-31+G* and B3LYP/6-31+G*//B3LYP-D3(BJ)/6$31+\mathrm{G}^{*}$ were found to differ only by $0.02 \mathrm{eV}$ in all cases. The stabilization of roughly $0.1 \mathrm{eV}$, gained in $[n] \mathrm{CPP}$ molecules with respect to previously calculated values, comes thus mainly from the dispersion-corrected geometries, since it has also been established before that the torsion between neighbouring benzene rings (higher here by $1-2^{\circ}$ upon inclusion of dispersion corrections) correlate with an increase of the HOMO-LUMO gap [59].

Summarizing, the overall effect of intra-molecular dispersion corrections is found to be small on individual molecules, but non-negligible if one searches the greatest possible accuracy. The effect is, as it was expected, more pronounced for the smallest nanorings, but we can generally conclude with respect to previously dispersion-uncorrected values that: (i) diameter (pore size) may decrease up to $0.1-0.4 \AA$ whereas dihedral angles between adjacent benzene rings may increase by $1-2^{\circ}$; (ii) strain energies decrease by $3-4 \%$ still following a strong decay with system size, and (iii) the HOMOLUMO gap decrease by $0.1 \mathrm{eV}$, which is however not expected to largely affect optoelectronic properties of these compounds.

\section{$3.2[6] \mathrm{CPP}$ as a case study}

The [6]CPP system is chosen next to further extend the study when going from isolated (gas-phase) to solvent- or solid-state phases. Note that this system is the only example, among the set of $[n]$ CPP compounds studied up to date, known to self-organize into completely linear, nanotube-like, struc- 
tures in the solid-state, which might pave the way towards the envisioned template-driven synthesis of SWNTs [5]. Therefore, the influence of intermolecular forces between weakly interacting molecules, arising from their self-aggregation in the solid-state, will be particularly challenging.

\subsubsection{Solvation phase}

The impact that solvation of [6]CPP molecules might have on their properties is approximately described here by using a dielectric continuum, the Polarizable Continuum Model (PCM) with the default technical parameters $[60,61]$ and for the dielectric constants $(\epsilon)$ corresponding to the set of solvents (of increasing dielectric force) used : $n$-hexane $(\epsilon=1.88)$, DMSO $(\epsilon=7.42)$, and THF $(\epsilon=46.8)$. The results in solution were obtained at the B3LYP-D3(BJ)/6-31+G* level with GAUSSIAN09 through the use of the implemented PCM continuum model. We note, related with the most remarkable properties studied before for the isolated system, that: (i) the pore size of [6]CPP does not change from gas- to solvent-phase, keeping a value of $8.38 \AA$ independently of the dielectric constant of the solvent; and (ii) the strain energy, $\Delta H_{\text {strain }}([6] \mathrm{CPP})$ as obtained from Eq. (5), only slightly changes from gas-phase $(89.83 \mathrm{kcal} / \mathrm{mol})$ to solution (e.g. $89.03 \mathrm{kcal} / \mathrm{mol}$ using THF as solvent). The small variation of these properties upon solvent polarity supports previous claims done in the literature, mainly based on isolated molecules $[59,62]$.

We have also observed small changes for the HOMO-LUMO gap, passing from $3.09 \mathrm{eV}$ (gas-phase) to 3.08, 3.05, and $3.04 \mathrm{eV}$, in $n$-hexane, DMSO, and THF, respectively. Actually, the strongest maximum absorption $\left(\lambda_{\max }\right)$ 
for this molecule does not originate from a HOMO to LUMO transition, but involves few transitions and peaks at around $340 \mathrm{~nm}$ independently of solvent effects [59]. Note that the vibronic effects on these optical properties have been recently investigated $[63,64]$. We have thus calculated, at the TimeDependent (TD) DFT level and with the same functional (B3LYP) and basis set $\left(6-31+\mathrm{G}^{*}\right)$ than those used before for obtaining the ground-state geometry, the TD-B3LYP/6-31+G* level, the lowest vertical absorption energy showing a non-negligible oscillator strength. In an attempt to disentangle the impact on the several concurring effects, we have systematically calculated the value of $\lambda_{\max }$ : (i) in gas-phase and at the ground-state geometry optimized without the dispersion correction; (ii) in gas-phase but employing dispersion-corrected ground-state geometries; and (iii) in solvent (with PCM) and at the latter dispersion-corrected geometries. The values found are 326,334 , and $343-345 \mathrm{~nm}$, respectively, with the latter values almost negligibly depending on the solvent polarity, are in close agreement with the experimental result.

\subsubsection{Interacting molecules}

The ORCA 3.0.0 package [65] was used for all calculations involving a discrete number of non-covalently bound molecules. The sequence of def2SVP (moderate), def2-TZVP (large) and def2-QZVP (very large) basis sets were used to largely (when the latter two are employed) reduce the Basis Set Superposition Error (BSSE) expected for binding energies of these non-covalently bound molecules. Thus, counterpoise correction to reduce the BSSE are not needed at this stage for DFT calculations. However, the numerical thresholds for integration grids, as well as for convergence of self- 
consistent calculations or optimizations, were always made tighter with respect to defaults, to reduce numerical errors when dealing with flat potential energy hypersurfaces or when accurate electronic densities were needed. Additionally, in the case of the larger systems tackled here, and again with the ORCA package, the computational effort was significantly reduced by invoking the 'resolution of the identity' (RI) [66] algorithm for Coulomb integrals, using for it the corresponding matching auxiliary basis sets [67].

The crystal packing of these molecules was recently resolved [21], leading to the discovery of a (somewhat unexpected) structural change, passing from a solid-state herringbone packing in the set of [8-12]CPP molecules to a linear alignment of $[6] \mathrm{CPP}$ molecules forming a discontinuous, nanotubelike, structure. This supramolecular organization might drive, for instance, a consistent bottom-up synthesis of a $(6,6)$ SWNT. To gain a further understanding of the strength and directionality of the underlying inter-molecular forces, as well as to investigate the energetical stability of these non-covalently bound dimers, we have resorted again to Eqs. (1)-(4), trying also to provide a robust answer to these questions independently of the method and/or correction employed. We are also aware that final crystalline structures may depend on other factors, such as difference in solvents or crystal packing forces, but estimating the strength and directionality of the underlying forces may nonetheless be of great interest.

We start by fully optimizing a dimer of [6]CPP molecules with the BLYPD3(BJ) method and the sequence of def2-SVP, def2-TZVP, and def2-QZVP basis sets. Figure 3 shows the optimized structure of this dimer, either for a lateral (top) or for a tubular (middle) alignments, as both are envisioned in 
real molecular samples (see Figure 4 for the arrangement of [6]CPP molecules in the crystalline state) [5]. The association (or binding) energy of every complex, which is known to be completely driven by non-covalent interactions, is calculated at this optimized structures by the expression:

$$
\Delta E_{\text {binding }}=E(\text { complex })-2 E(\text { monomer }),
$$

where dispersion-corrected energies are consequently used thereof for both the complex, [6]CPP $\cdots[6] \mathrm{CPP}$, and the monomer, a single [6]CPP molecule. We take first the lateral structure as example for further discussion and benchmarking, with the calculated values for $\Delta E_{\text {binding }}$ being $-9.33,-7.57$, and $-7.33 \mathrm{kcal} / \mathrm{mol}$ at the BLYP-D3(BJ)/def2-SVP, BLYP-D3(BJ)/def2TZVP, and BLYP-D3(BJ)/def2-QZVP levels, respectively. We note the (expectedly large) BSSE when using the smallest (def2-SVP) basis set, and how this error is practically negligible with the (large) def2-TZVP extension (around $0.2 \mathrm{kcal} / \mathrm{mol}$ with respect to the nearly-converged def2-QZVP value). Interestingly, the BLYP-D3(BJ)/def2-TZVP method gives for the tubular structure a value for $\Delta E_{\text {binding }}$ of $-12.95 \mathrm{kcal} / \mathrm{mol}$, which is 1.7 times larger than the corresponding for the lateral case.

However, the use of large basis sets (such as def2-TZVP and beyond) might hamper the study of larger nanoaggregates of [6]CPP molecules if needed. This is why we apply next, and to the lateral case again, the less costly HF-3c method, noticing for fully optimized geometries that: (i) the pore size of a $[6] \mathrm{CPP}$ monomer amounts to $8.36 \AA$, and it is thus close to the value (8.39 A) obtained before with the BLYP-D3(BJ)/def2-QZVP method; and (ii) the $\Delta E_{\text {binding }}$ value is now $-7.26 \mathrm{kcal} / \mathrm{mol}$, being also very close to the best value $(-7.33 \mathrm{kcal} / \mathrm{mol})$ obtained with the BLYP-D3(BJ)/def2-QZVP method. These results seem to render the HF-3c method as very accurate 
for further studies of large systems, as it has been also recently highlighted for the refinement of protein structures [68]. As a matter of example, the tubular alignment for a dimer of [6]CPP molecules is now predicted to have a $\Delta E_{\text {binding }}$ value of $-12.36 \mathrm{kcal} / \mathrm{mol}$, and again approximately twice as large as that for the lateral arrangement studied before, which also points to a possible microscopic epitaxy-like growth mechanism.

We can therefore afford with the HF-3c method the study of more extended (e.g. lateral trimer and tetramer) fully optimized packing forms (see the bottom structure displayed in Figure 3) thanks to the excellent trade-off found between accuracy and computational cost. Actually, the $\Delta E_{\text {binding }}$ energy is calculated now to be -19.48 and $-27.32 \mathrm{kcal} / \mathrm{mol}$, for the trimer and tetramer, respectively. We additionally apply a three-body correction, see Eq. (2), to account for part of the still missing many-body effects $[69,70]$, which is repulsive and amounts to 0.89 and $1.51 \mathrm{kcal} / \mathrm{mol}$ for the trimer and the tetramer, respectively, leading to final estimates of -18.59 and and $-25.81 \mathrm{kcal} / \mathrm{mol}$. If we divide now this association energy by the number of interacting pairs of dimers, the strength of the dimer interactions can be estimated, with due caution, to be -6.20 and $-5.16 \mathrm{kcal} / \mathrm{mol}$, respectively. We want to note in passing that the energies are now lower than those calculated for an isolated dimer since the latter misses some many-body effects. However, if we apply the BLYP-NL/def2-QZVP method, at the HF-3c optimized structure for a dimer, we find an energy of $-5.81 \mathrm{kcal} / \mathrm{mol}$. The above results point towards a $\Delta E_{\text {binding }} \simeq 5-6 \mathrm{kcal} / \mathrm{mol}$ as a rough estimate for a dimer of lateral molecules within the same layer of the crystal structure; and further legitimate the conclusions dropped before about the larger stability of the tubular arrangement. 


\subsubsection{Crystalline structure}

The crystalline structure is necessarily more involved due the large set of existing intra-layer and inter-layer (dimer) interactions. One can calculate the lattice or cohesive energy through the set of unique dimer interactions within the unit cell [71-73]. Structural data for the unit cell, as taken from the Cambridge Crystallographic Data Centre (code archive CCDC 852989), were used rigidly herein. Figure 4 shows that in this case they reduce to the three pairs named here as lateral (i.e. two adjacent molecules belonging to the same layer and interacting along the $a, b$ directions), tubular-like (i.e. two adjacent and superimposed molecules belonging to different layers and interacting along the $c$ axis), and diagonal (i.e. two molecules belonging to different layers but not superimposed). In this case, the cohesive energy is given by:

$$
E_{\text {cohesive }}=\frac{1}{2} \sum_{i}^{\text {dimers }} n_{i} \Delta E_{\text {binding }}^{(i)}
$$

where $n_{i}$ refers to the number of symmetry-related pairs, with their corresponding binding energy calculated by the HF-3c method. The result must be divided by two due to the counting method used, see Ref. [74] for further details. We obtain $\Delta E_{\text {binding }}$ values (in $\left.\mathrm{kcal} / \mathrm{mol}\right)$ of $-7.23\left(n_{i}=6\right),-12.92$ $\left(n_{i}=2\right)$, and $-3.74\left(n_{i}=12\right)$ for the lateral, tubular-like, and diagonal packing motifs, respectively, which we use next to feed Eq. (7), yielding a cohesive energy of $-57.0 \mathrm{kcal} / \mathrm{mol}$.

We now proceed to compare the above estimate with that obtained by dispersion-corrected DFT calculations using periodic boundary conditions 
(PBC) $[75-77]$ as:

$$
E_{\text {cohesive }}=\frac{E_{\text {bulk }}}{Z}-E_{\text {molecule }}
$$

where $E_{b u l k}$ is the total energy of a unit cell $(a=b=19.3957 \AA, c=6.1998 \AA$, $\alpha=\beta=90^{\circ}, \gamma=120^{\circ}$ ), including $Z$ molecules, and $E_{\text {molecule }}$ the energy of an isolated molecule, both energies calculated here at the dispersion-corrected BLYP-D2 level. The ABINIT code [78-80] was employed for the calculations we report next. The technical details for these calculations are briefly presented next for clarity: (i) the norm-conserving Martins-Troullier pseudopotentials of the extended Car-Parrinello molecular dynamics pseudolibrary (http://cpmd.org), converted to the FHI format, were employed [81]; (ii) a cutoff energy of plane waves was fixed at 100Ry after checking for convergence of values up to $0.1 \mathrm{~kJ} / \mathrm{mol}$; (iii) the Monkhorst-Pack scheme was used to sample the Brillouin zone, with a $k$-point grid of $2 \times 2 \times 6$ checked also for convergence with respect to the corresponding $3 \times 3 \times 6$ grid; and (iv) the energy of the corresponding monomer was obtained at the $\Gamma$ point using a cell of dimensions $35 \times 35 \times 20 \AA$ alongside the $x, y$, and $z$ directions, respectively, checked also for convergence with respect to a cell of dimensions 40x40x25 $\AA$. This leads to a calculated cohesive energy of $-47.6 \mathrm{kcal} / \mathrm{mol}$, slightly lower than that calculated before at the HF-3c level from pair interactions.

Nonetheless, the agreement found between the two values is quite remarkable, taking into account the strong differences between both computational strategies. This allows us to consider a value around $50 \mathrm{kcal} / \mathrm{mol}$ as a first estimate of the $[6] \mathrm{CPP}$ cohesive energy. These results indicate also that the computationally cheap HF-3c method provides accurate-enough values, in agreement with previous findings for the X23 dataset of molecular crystals [82], and can be used for instance to quantitatively bracket the cohesive 
energies or to rapidly screen larger systems. Finally, the PBC-BLYP-D2 value is made positive and further used to estimate a sublimation enthalpy (at the room temperature of $298.15 \mathrm{~K}$ and not yet measured [83]) of 42.6 $\mathrm{kcal} / \mathrm{mol}$ by using the expression $\Delta_{s} H(T)=-E_{\text {cohesive }}-2 R T$, with $2 R T$ being the thermal contribution to be included [84-88].

\section{Concluding remarks}

In summary, we have theoretically studied first a set of intra-molecular properties of increasingly larger $[n] \mathrm{CPP}(n=4-12)$ systems to better understand the influence of the dispersion interactions, as a function of the system size. Despite the fact that the diameter of the macrocycle increases with $n$, and thus the face-to-face interactions between atoms belonging to opposite benzene rings are reduced due to the decay with their increasing distance, these macrocycles still hold large interactions between adjacent benzene rings. Hence, these interactions are found to slightly affect all kind of properties of isolated molecules, such as the diameter of the macrocycle, the dihedral angles between adjacent benzene rings, the strain energy to close the chain into a macrocycle, or the frontier orbital energies and their corresponding energy difference.

Then, in a further step, we have thoroughly extended the study to the self-aggregation of [6]CPP molecules, which is known to form discontinuous nanotube-like structures upon crystallization. We have systematically studied a dimer $([6] \mathrm{CPP} \cdots[6] \mathrm{CPP})$ in the two main arrangements found in the solid-state: either with the two macrocycles being lateral or with one on top of the another. Our results have shown a much larger energy stability, driven 
completely by non-covalent interactions, of the latter structure with respect to the former, which might help to understand the mechanism followed by these molecules upon crystallization.

Besides this, with the help of the whole set of results for the unique dimer packing motifs, along several directions of the crystalline lattice, we have calculated the (strong) cohesive energy of the [6]CPP crystals. The seemingly large value found has been further confirmed using periodic boundary conditions, giving us confidence in the value obtained.

We hope these results will help to understand better the rich properties of these compounds, as well as to pave the way towards further theoretical studies of self-aggregation or inclusion compounds formed by these fascinating molecules.

\section{Acknowledgements}

This work is supported by the "Ministerio de Economia y Competitividad" of Spain and the "European Regional Development Fund" through projects CTQ2014-55073-P and FIS2012-35880.

\section{References}

[1] H.-B. Li, A. J. Page, S. Irle, K. Morokuma, ChemPhysChem 2012, 13, 1479 .

[2] H. Omachi, T. Nakayama, E. Takahashi, Y. Segawa, K. Itami, Nat. Chem. 2013, 5, 572 . 
[3] P. E. Evans, R. Jasti, Top. Curr. Chem. 2014, 349, 249.

[4] P. E. Evans, E. R. Darzi, R. Jasti, Nat. Chem. 2014, 6, 404.

[5] E. S. Hirst, R. Jasti, J. Org. Chem. 2012, 77, 10473.

[6] N. Toriumi, A. Muranaka, E. Kayahara, S. Yamago, M. Uchiyama, J. Am. Chem. Soc. 2015, 137, 82.

[7] P. J. Evans, R. Jasti, Top. Curr. Chem. 2012, 128, 415.

[8] S. Yamago, Y. Watanabe, T. Iwamoto, Angew. Chem. Int. Ed. 2011, 50, 3244 .

[9] Y. Segawa, H. Omachi, K. Itami, Org. Lett. 2010, 12, 2262.

[10] S. Bachrach, D. Stück, J. Org. Chem. 2010, 75, 6595.

[11] T. Iwamoto, Y. Watanabe, Y. Sakamoto, T. Suzuki, S. Yamago, J. Am. Chem. Soc. 2011, 133, 8354.

[12] S. Hitosugi, W. Nakanishi, T. Yamasaki, H. Isobe, Nat. Commun. 2011, 2, 492 .

[13] K. Itami, Pure Appl. Chem. 2012, 84, 907.

[14] E. Kayahara, T. Iwamoto, T. Suzuki, S. Yamago, Chem. Lett. 2013, 42, 621.

[15] M. Fujitsuka, S. Tojo, T. Iwamoto, E. Kayahara, S. Yamago, T. Majima, J. Phys. Chem. Lett. 2014, 5, 2302.

[16] E. Kayahara, V. K. Patel, S. Yamago, J. Am. Chem. Soc. 2014, 136, 2284 . 
[17] S. Yamago, E. Kayahara, T. Iwamoto, Chem. Rec. 2014, 14, 84.

[18] T. J. Jisto, X. Xian, R. Jasti, J. Org. Chem. 2012, 77, 5857.

[19] R. Jasti, J. Bhattacharjee, J. N. Neaton, C. R. Bertozzi, J. Am. Chem. Soc. 2008, 130, 17646.

[20] T. Iwamoto, Y. Watanabe, T. Sadahiro, T. Haino, S. Yamago, Angew. Chem. Int. Ed. 2011, 50, 8342.

[21] J. Xia, J. W. Bacon, R. Jasti, Chem. Sci. 2012, 3, 3018.

[22] T. Iwamoto, Y. Watanabe, H. Takaya, T. Haino, N. Yasuda, S. Yamago, Chem. Eur. J. 2013, 19, 14061.

[23] T. Iwamoto, Z. Slanina, N. Mizorogi, J. Guo, T. Akasaka, S. Nagase, H. Takaya, N. Yasuda, T. Kato, S. Yamago, Chem. Eur. J. 2014, 20, 14403.

[24] H. Omachi, Y. Segawa, K. Itami, Org. Lett. 2011, 13, 2480.

[25] A. Yagi, Y. Segawa, K. Itami, J. Am. Chem. Soc. 2012, 134, 2962.

[26] J. Xia, M. R. Golder, M. E. Foster, B. M. Wong, R. Jasti, J. Am. Chem. Soc. 2012, 134, 19709.

[27] S. Grimme, WIREs Comput. Mol. Sci. 2011, 1, 211.

[28] S. Grimme, M. Steinmetz, Phys. Chem. Chem. Phys. 2013, 15, 16031.

[29] P. Hohenberg, W. Kohn, Phys. Rev. B 1964, 136, 864.

[30] W. Kohn, L. J. Sham, Phys. Rev. A 1965, 140, 1133.

[31] P. Jurečka, J. Sponer, J. Černy, P. Hobza, Phys. Chem. Chem. Phys. 2006, 8, 1985. 
[32] J. Č́rny, P. Hobza, Phys. Chem. Chem. Phys. 2007, 9, 5291.

[33] K. E. Riley, M. Pitoňák, P. Jurečka, P. Hobza, Chem. Rev. 2010, 110, 5023.

[34] A. D. Becke, Phys. Rev. A 1988, 38, 3098.

[35] C. Lee, W. Yang, R. G. Parr, Phys. Rev. B 1988, 37, 785.

[36] A. D. Becke, J. Chem. Phys. 1993, 98, 5648.

[37] V. Barone, C. Adamo, Chem. Phys. Lett. 1994, 224, 432.

[38] P. J. Stephens, F. J. Devlin, C. F. Chabalowski, M. J. Frisch, J. Phys. Chem. 1994, 98, 11623.

[39] F. Weigend, R. Ahlrichs, Phys. Chem. Chem. Phys. 2005, 7, 3297.

[40] V. V. Gobre, A. Tkatchenko, Nature Commun. 2013, 4, 2341.

[41] A. Ambrosetti, D. Alfè, R. A. DiStasio Jr, A. Tkatchenko, J. Phys. Chem. Lett. 2014, 5, 849.

[42] S. Grimme, J. Antony, S. Ehrlich, H. Krieg, J. Chem. Phys. 2010, 132, 154104 .

[43] S. Grimme, S. Ehrlich, L. Goerigk, J. Comput. Chem. 2011, 32, 1456.

[44] A. D. Becke, E. R. Johnson, J. Chem. Phys. 2005, 122, 154101.

[45] E. R. Johnson, A. D. Becke, J. Chem. Phys. 2005, 123, 024101.

[46] E. R. Johnson, A. D. Becke, J. Chem. Phys. 2006, 124, 174104.

[47] S. Grimme, J. Comput. Chem. 2006, 27, 1787. 
[48] P. Jurečka, J. Černý, P. Hobza, D. R. Salahub, J. Comput. Chem. 2007, 28,555 .

[49] O. A. von Lilienfeld, A. Tkatchenko, J. Chem. Phys. 2010, 132, 234109.

[50] M. R. Kennedy, A. R. McDonald, A. E. DePrince III, M. S. Marshall, R. Podeszwa, C. David Sherrill, J. Chem. Phys. 2014, 140, 121104.

[51] O. A. Vydrov, T. Van Voorhis, J. Chem. Phys. 2010, 133, 244103.

[52] W. Hujo, S. Grimme, J. Chem. Theory Comput. 2011, 7, 3866.

[53] W. Hujo, S. Grimme, J. Chem. Theory Comput. 2013, 9, 308.

[54] R. Sure, S. Grimme, J. Comput. Chem. 2013, 34, 1672.

[55] M. J. Frisch, G. W. Trucks, H. B. Schlegel, G. E. Scuseria, M. A. Robb, J. R. Cheeseman, G. Scalmani, V. Barone, B. Mennucci, G. A. Petersson, H. Nakatsuji, M. Caricato, X. Li, H. P. Hratchian, A. F. Izmaylov, J. Bloino, G. Zheng, J. L. Sonnenberg, M. Hada, M. Ehara, K. Toyota, R. Fukuda, J. Hasegawa, M. Ishida, T. Nakajima, Y. Honda, O. Kitao, H. Nakai, T. Vreven, J. A. Montgomery, Jr., J. E. Peralta, F. Ogliaro, M. Bearpark, J. J. Heyd, E. Brothers, K. N. Kudin, V. N. Staroverov, R. Kobayashi, J. Normand, K. Raghavachari, A. Rendell, J. C. Burant, S. S. Iyengar, J. Tomasi, M. Cossi, N. Rega, J. M. Millam, M. Klene, J. E. Knox, J. B. Cross, V. Bakken, C. Adamo, J. Jaramillo, R. Gomperts, R. E. Stratmann, O. Yazyev, A. J. Austin, R. Cammi, C. Pomelli, J. W. Ochterski, R. L. Martin, K. Morokuma, V. G. Zakrzewski, G. A. Voth, P. Salvador, J. J. Dannenberg, S. Dapprich, A. D. Daniels, Ö. Farkas, J. B. Foresman, J. V. Ortiz, J. Cioslowski, and D. J. Fox, "Gaussian 09 Revision D.01" (2009), gaussian Inc. Wallingford CT. 
[56] J.C. Sancho-García, J. Cornil, J. Chem. Theor. Comput. 2005, 1, 581.

[57] S. E. Wheleer, WIREs Comput. Mol. Sci. 2012, 2, 204.

[58] M. Fujitsuka, T. Iwamoto, E. Kayahara, S. Yamago, T. Majima, ChemPhysChem 2013, 14, 1570.

[59] Y. Segawa, A. Fukuzawa, S. Matsuura, H. Omachi, S. Yamaguchi, S. Irle, K. Itami, Org. Biomol. Chem. 2012, 10, 5979.

[60] J. Tomasi, B. Mennucci, R. Cammi, Chem. Rev. 2005, 105, 2999.

[61] G. Scalmani, M. J. Frisch, J. Chem. Phys. 2010, 132, 114110.

[62] P. Li, T. J. Sisto, E. R. Darzi, R. Jasti, Org. Lett. 2014, 16, 182.

[63] C. Camacho, T. A. Niehaus, K. Itami, S. Irle, Chem. Sci. 2013, 4, 187.

[64] V. S. Reddy, C. Camacho, J. Xia, R. Jasti, S. Irle, J. Chem. Theory Comput. 2014, 10, 4025.

[65] F. Neese, WIREs Comput. Mol. Sci. 2012, 2, 73.

[66] K. Eichkorn, O. Trutler, H. Öhm, M. Häser, R. Ahlrichs, Chem. Phys. Lett. 1995, 240, 283.

[67] K. Eichkorn, F. Weigend, O. Trutler, R. Ahlrichs, Theor. Chem. Acc. 1997, 97, 119 .

[68] L. Goerigk, C. A. Collyer, J. R. Reimers, J. Phys. Chem. B 2014, 118, 14612.

[69] A. Tkatchenko, R. A. DiStasio, R. Car, M. Scheffler, Phys. Rev. Lett. 2012, 108, 236402 . 
[70] R. A. DiStasio, A. O. von Lilienfeld, A. Tkatchenko, Proc. Natl. Acad. Sci. USA 2012, 109, 14791.

[71] A. L. Ringer, C. D. Sherrill, Chem. Eur. J. 2008, 14, 2542.

[72] G. J. O. Beran, K. Nanda, J. Phys. Chem. Lett. 2010, 1, 3480.

[73] J. C. Sancho-García, J. Aragó, E. Ortí, Y. Olivier, J. Chem. Phys. 2013,138, 204304.

[74] W. B. Schweizer, J. B. Dunitz, J. Chem. Theory Comput. 2006, 2, 288.

[75] A. M. Reilly, A. Tkatchenko, J. Chem. Phys. 2013, 139, 024705.

[76] J. Moellmann, S. Grimme, J. Phys. Chem. C 2014, 118, 7615.

[77] L. Kronik, A. Tkatchenko, Acc. Chem. Res. DOI:10.1021/ar500144s

[78] F. Bottin, S. Leroux, A. Knyazev, G. zerath, Comput. Mat. Science 2008, 42, 329 .

[79] X. Gonze, B. Amadon, P.-M. Anglade, J.-M. Beuken, F. Bottin, P. Boulanger, F. Bruneval, D. Caliste, R. Caracas, M. Cote, T. Deutsch, L. Genovese, Ph. Ghosez, M. Giantomassi, S. Goedecker, D. R. Hamann, P. Hermet, F. Jollet, G. Jomard, S. Leroux, M. Mancini, S. Mazevet, M. J. T. Oliveira, G. Onida, Y. Pouillon, T. Rangel, G.-M. Rignanese, D. Sangalli, R. Shaltaf, M. Torrent, M. J. Verstraete, G. Zerah, J. W. Zwanziger, Computer Phys. Commun. 2009, 180, 2582.

[80] M.A.L. Marques, M.J.T. Oliveira, T. Burns, Comput. Phys. Commun. 2012, 183, 2227.

[81] M. Boero, M. Parrinello, K. Terakura, J. Am. Chem. Soc. 1998, 120, 2746 . 
[82] J. G. Branderburg, S. Grimme, Top. Curr. Chem. 2014, 345, 1.

[83] M. V. Roux, M. Temprano, J. S. Chickos, Y. Nagano, J. Phys. Chem. Ref. Data 2008, 37, 1855.

[84] J. S. Chickos, W. E. Acree, Jr., J. Phys. Chem. Ref. Data 2002, 31, 537.

[85] I. A. Fedorov, Y. N. Zhuravlev, V. P. Berveno, Phys. Chem. Chem. Phys. 2011, 13, 5679 .

[86] A. Otero-de-la-Roza, E. R. Johnson, J. Chem. Phys. 2012, 137, 054103.

[87] I. A. Fedorov, Y. N. Zhuravlev, V. P. Berveno, J. Chem. Phys. 2013, 138, 094509 .

[88] Y. Huang, Y. Shao, G. J. O. Beran, J. Chem. Phys. 2013, 138, 224112. 
- Table 1. Summary of main computational details employed in the study.

- Table 2. Evolution of the diameter $(d$, in $\AA$ ) of increasingly longer $[n] \mathrm{CPP}$ compounds, as calculated at the B3LYP-D3(BJ)/6-31+ $\mathrm{G}^{*}$ level. The difference with respect to previously reported B3LYP/6-31G* dispersionuncorrected values is shown in parentheses.

- Table 3. Evolution of strain energy $\left(\Delta H_{\text {strain }}\right.$, in kcal $\left./ \mathrm{mol}\right)$ and HOMOLUMO energy difference $(\Delta \epsilon$, in $\mathrm{eV})$ of increasingly longer $[n] \mathrm{CPP}$ compounds, as calculated at the B3LYP-D3(BJ)/6-31+G* level. 
Table 1:

\begin{tabular}{|c|c|c|c|c|c|}
\hline Property & Method & $\begin{array}{l}\text { Dispersion } \\
\text { correction }\end{array}$ & $\begin{array}{c}\text { Basis } \\
\text { sets }\end{array}$ & $\begin{array}{l}\text { Software } \\
\text { package }\end{array}$ & Release \\
\hline$d, \mathrm{C}_{o}-\mathrm{C}_{i p s o^{-}}-\mathrm{C}_{i p s o^{\prime}}-\mathrm{C}_{o^{\prime}}$ & B3LYP & -D3(BJ) & $6-31+\mathrm{G}^{*}$ & GAUSSIAN09 & D.01 \\
\hline$\Delta H_{\text {strain }}$ & B3LYP & -D3(BJ) & $6-31+\mathrm{G}^{*}$ & GAUSSIAN09 & D.01 \\
\hline \multirow[t]{3}{*}{$\Delta E_{\text {binding }}$} & BLYP & -D3(BJ) & def2-TZVP/def2-QZVP & ORCA & 3.0 .0 \\
\hline & & $-\mathrm{NL}$ & def2-TZVP/def2-QZVP & ORCA & 3.0 .0 \\
\hline & $\mathrm{HF}-3 \mathrm{c}$ & -D3(BJ) & - & ORCA & 3.0 .0 \\
\hline \multirow[t]{2}{*}{$E_{\text {cohesive }}$} & BLYP & $-\mathrm{D} 2$ & plane waves/pseudopotentials & ABINIT & 7.4 .3 \\
\hline & $\mathrm{HF}-3 \mathrm{c}$ & -D3(BJ) & - & ORCA & 3.0 .0 \\
\hline
\end{tabular}


Table 2:

\begin{tabular}{rc}
\hline$n$ & $d$ \\
\hline 4 & $5.68(-0.02)$ \\
5 & $6.67(-0.38)$ \\
6 & $8.38(-0.02)$ \\
7 & $9.48(-0.30)$ \\
8 & $11.10(-0.02)$ \\
9 & $12.32(-0.17)$ \\
10 & $13.85(-0.02)$ \\
11 & $15.12($ n.a. $)$ \\
12 & $16.58(-0.02)$ \\
\hline
\end{tabular}


Table 3:

\begin{tabular}{rrr}
\hline$n$ & $\Delta H_{\text {strain }}$ & $\Delta \epsilon$ \\
\hline 4 & 134.12 & 2.47 \\
5 & 108.58 & 2.60 \\
6 & 89.80 & 3.07 \\
7 & 79.16 & 3.11 \\
8 & 67.77 & 3.35 \\
9 & 61.91 & 3.35 \\
10 & 54.25 & 3.49 \\
11 & 50.72 & 3.48 \\
12 & 45.15 & 3.57 \\
\hline
\end{tabular}


- Figure 1. Chemical structure of a model armchair SWNT (left) and that of the investigated $[n] \mathrm{CPP}$ compounds (right). The hydrogen atoms and corresponding $\mathrm{C}-\mathrm{H}$ bonds have been omitted for clarity.

- Figure 2. From top to bottom: (i) Evolution of strain energies with the number $(n)$ of rings in $[n] \mathrm{CPP}$ compounds; (ii) evolution of energy difference between the frontier orbitals with the number $(n)$ of rings in $[n]$ CPP compounds.

- Figure 3. From top to bottom: Optimized structure of a dimer in both (a) lateral and (b) tubular-like views, and of a (c) tetramer of [6]CPP molecules.

- Figure 4. Packing arrangement of [6]CPP in the crystalline state. 

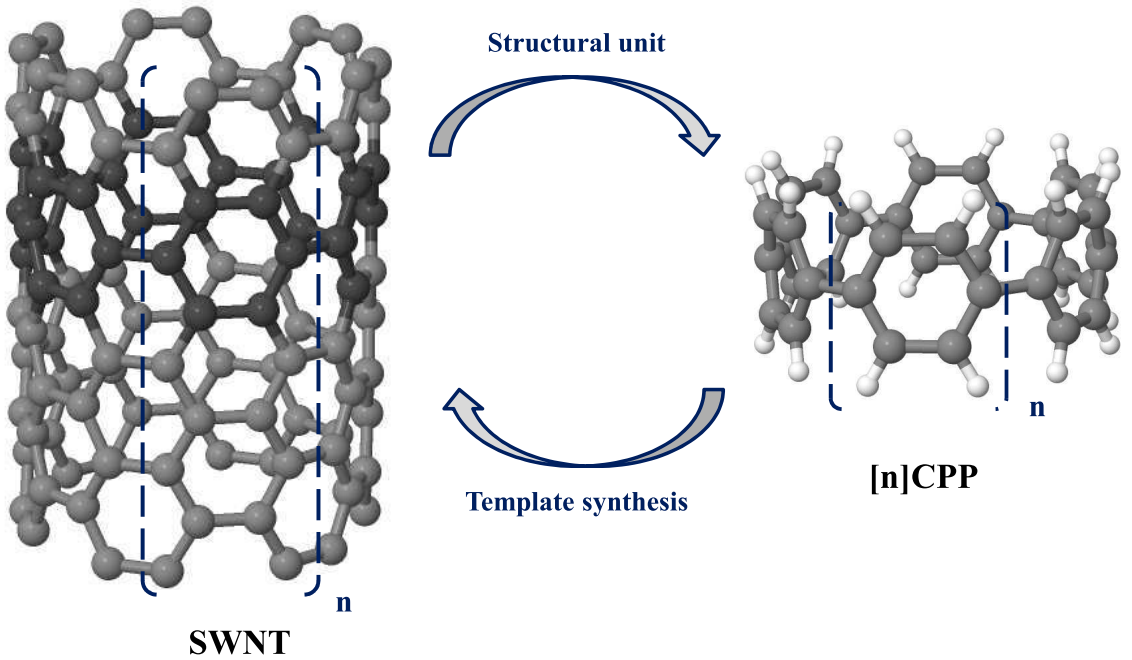

Figure 1. 

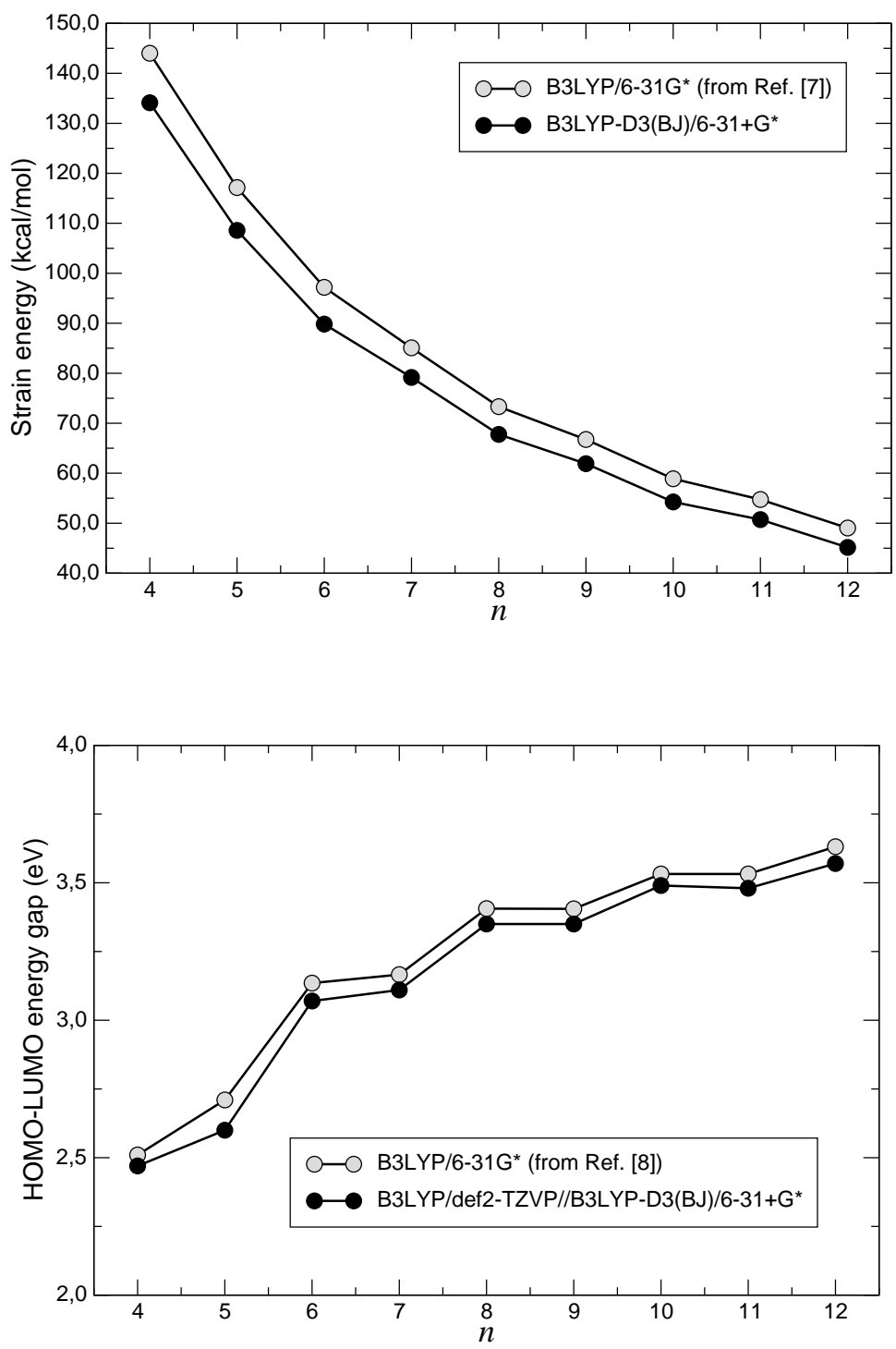

Figure 2. 
(a)
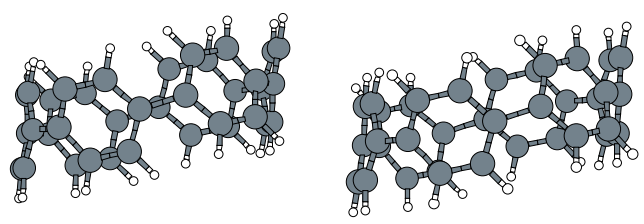

(b)
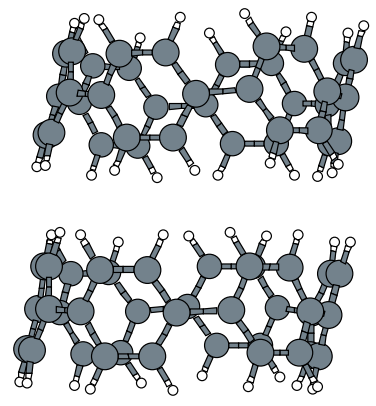

(c)

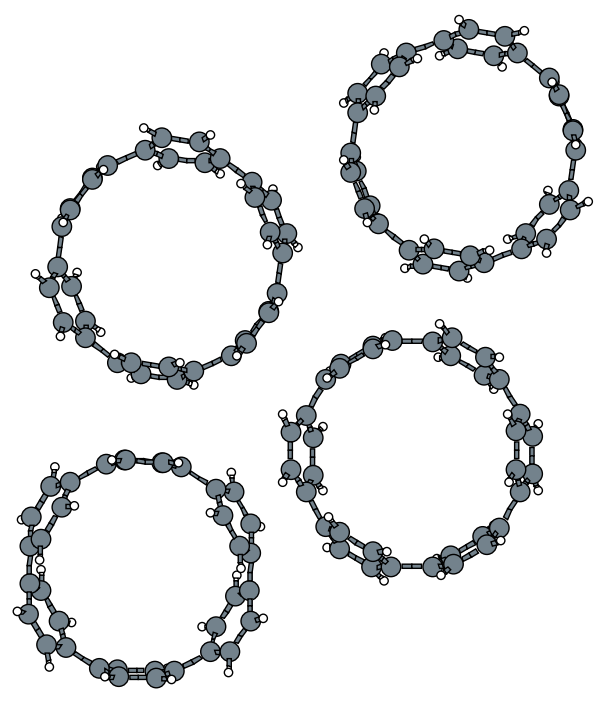

Figure 3. 


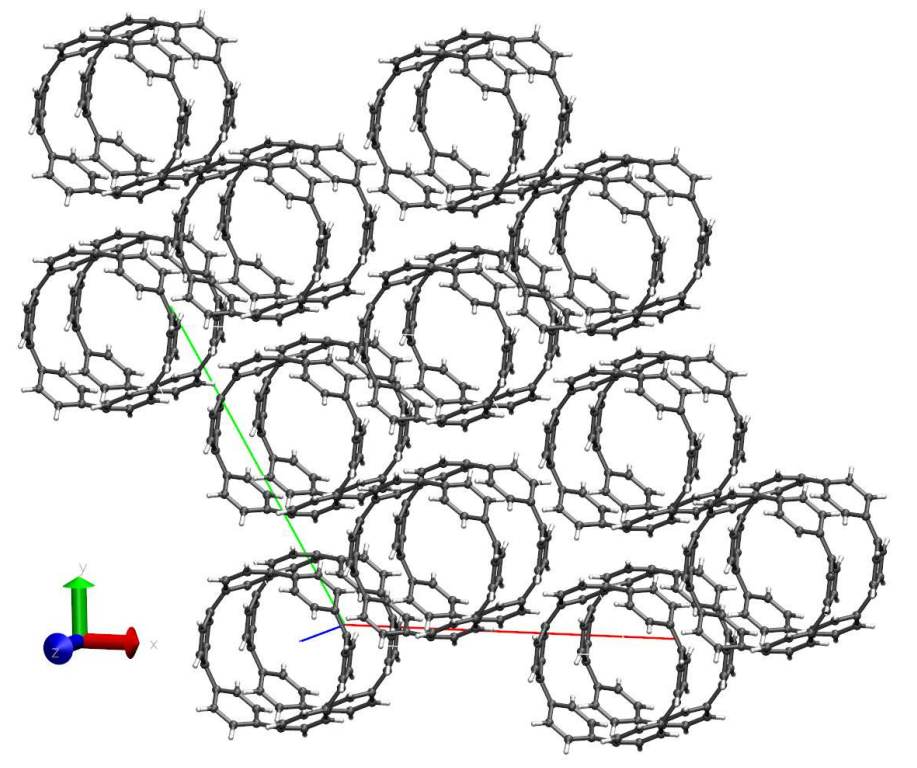

Figure 4. 